\title{
Algorithms for synthesis of a fuzzy control system chemical reactor temperature
}

\author{
Isamiddin Siddikov ${ }^{1}$, Nodira Mamasodikova ${ }^{2}$, Odinaxon Rayimdjanova ${ }^{3}$, Davronbek \\ Khalmatov ${ }^{3}$ and Xuryat Mirzaaxmedova ${ }^{3}$ \\ ${ }^{1}$ Tashkent State Technical University, 2, Universitetskaya street, Tashkent, 100095, Uzbekistan \\ ${ }^{2}$ Fergana branch of Tashkent University of Information Technologies named after Mukhammad al-Khwarizmi, \\ 185, Mustaqillik street, Fergana, 150118, Uzbekistan \\ ${ }^{3}$ Tashkent Institute of Textile and Light Industry, 5, Shakhdjakhon street, Tashkent, 100100, Uzbekistan
}

\begin{abstract}
The issues of synthesis of a fuzzy control system for ill-defined technological processes are considered. An effective algorithm for the synthesis of a fuzzy logic controller and a fuzzy system for automatic regulation of the temperature regime of a chemical reactor, invariant to parametric and external disturbances, is presented. The proposed synthesis algorithm for a fuzzy-logical proportional-integral-differential (PID) -controller is simple and allows you to use a standard form of description of linguistic variables and a minimum set of control rules. The synthesized fuzzy logic controller gives the entire automatic control system the ability to maintain the reactor temperature at a given level in the presence of external disturbances, as well as to qualitatively control the technological process with a wide range of changes in its parameters over time. The used methods of the theory of fuzzy logic and neural networks allow you to operate with linguistic fuzzy statements. The bases of the rules of logical inference of a fuzzy-logical regulator in the form of a Cartesian product of fuzzy sets with a membership function, which has a trapezoidal shape, have been formed. The results of modeling a fuzzylogic control system showed that if there is a noisy external disturbing signal in the system and its level changes up to $30 \%$, as well as changes in the parameters of the control object (gain and constant time) up to $25 \%$ (in the direction of increasing and decreasing), the fuzzy system retains the properties of stability.
\end{abstract}

\section{Keywords}

Algorithm, synthesis, fuzzy controller, chemical reactor, uncertainty, linguistic variable, control system

\section{Introduction}

Analysis of the state of the problem of designing control systems for complex technological objects shows that traditional methods of constructing models of objects and control systems for them do not lead to satisfactory results when the initial description of the problem to be solved is obviously inaccurate and incomplete $[1,2,7,9]$.

As a rule, these poorly structured or poorly defined objects have such properties as non-stationarity of parameters, incomplete information about objects, lack of a formal description of the control object, etc. $[1,6,8,12]$. From the point of view of the classical theory of automatic control (ACA), the control of objects of this class is a rather complicated, in most cases unsolvable problem. This is due to the fact that when building a traditional control system (CS), it is necessary to formally describe the control

\footnotetext{
III International Workshop on Modeling, Information Processing and Computing (MIP: Computing-2021), May 28, 2021, Krasnoyarsk, Russia

EMAIL: isiddikov54@gmail.com (Isamiddin Siddikov); nodiramamasodikova@mail.ru (Nodira Mamasodikova); rodinaxon75@mail.ru (Odinaxon Rayimdjanova); holdav@mail.ru (Davronbek Khalmatov); mirzaaxmedova1961@gmail.com (Xuryat Mirzaaxmedova) ORCID: 0000-0001-9681-5156 (Isamiddin Siddikov); 0000-0002-7605-879X (Nodira Mamasodikova); 0000-0003-0333-7357 (Odinaxon Rayimdjanova); 0000-0002-2120-6257 (Davronbek Khalmatov); 0000-0002-2933-064X (Xuryat Mirzaaxmedova)

(C) (i) (C) 2021 Copyright for this paper by its authors.

(c) Use permitted under Creative Commons License Attribution 4.0 International (CC BY 4.0).

[CEUR Workshop Proceedings (CEUR-WS.org)
} 
object in advance and form control criteria on the basis of a certain mathematical apparatus operating in quantitative categories.

If it is impossible to give an exact mathematical description of the object and its control criteria in quantitative terms, the traditional control theory turns out to be inapplicable $[4,11,13,15]$. It is in these cases that it is advisable to use intelligent control methods to solve the problem of creating a control system specifically focused on building models that take into account the incompleteness and inaccuracy of the initial data.

\section{Formulation of the problem}

The processes taking place in the reactor depend on the temperaturein the reactor, the percentage of all components and the flow rate of the reaction mixture. The main input parameters of this process are: steam and demineralized water consumption; initial temperature in jacket and reactor; initial concentration of the components of the reaction mixture. The rest of the influences are disturbing; the pressure of the heating steam can be taken as the main disturbing effect.

Under certain assumptions and on the basis of the material balance equations $[3,8]$, the structuralfunctional model of a chemical reactor with a steam jacket can be represented in the following form (Figure 1:):

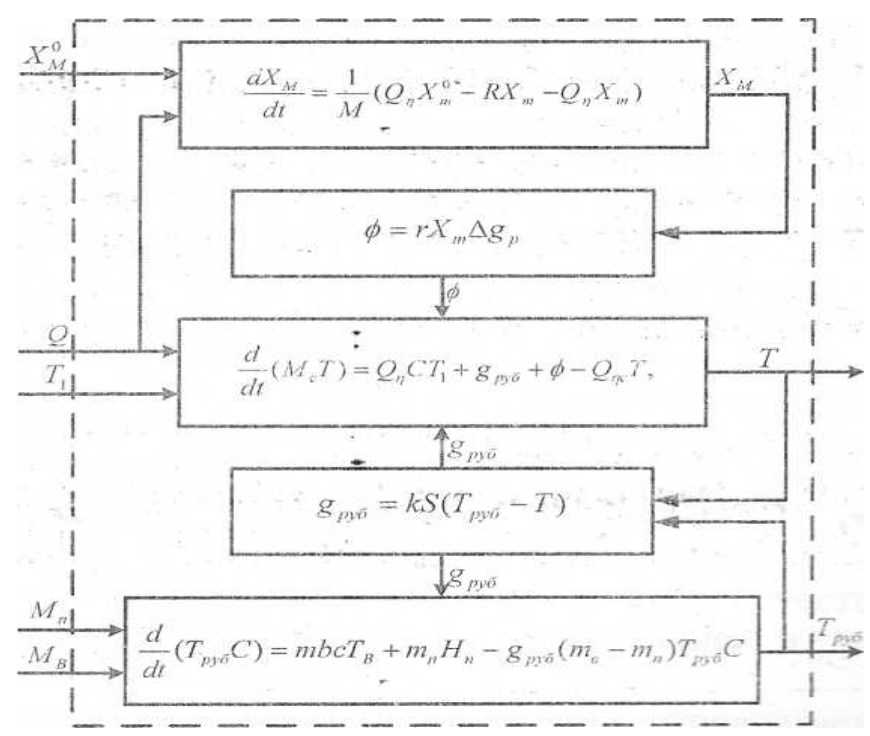

Figure 1: Structural and functional model of a chemical reactor with a steam jacket

Where $M$ is the mass of the contents of the reactor; $M_{c}$ - the mass of the reaction mixture; $X_{m}$ the concentration of monomers in the reactor; $Q_{1}$ - the flow rate of the reaction mixture at the entrance to the reactor; $Q_{2}$ - the flow rate of the output stream; $X_{m}^{0}$ - the concentration of monomers; $R$ constant of the reaction rate; $\Delta g_{p}$ - the thermal effect of the reaction; $C$ - heat capacity of the contents of the reactor; $\mathrm{T}_{\text {shi- }}$ temperature in the shirt; $T$ - reactor temperature; $T_{B}$ - water temperature; $H_{n}$ enthalpy of steam; $S$ - the surface area of the jacket heat exchange; $g_{s h i}$ heat flow from the jacket; $k$ - coefficient of heat transfer from the jacket to the reactor; $\phi$ - heat generated; $m_{b}$ - consumption of water supplied to the jacket; $m_{n}$ - steam consumption at the jacket inlet.

The input control effect for the reactor temperature is the heating steam consumption, and the rest of the influences are disturbing [3,9].

One of the most important parameters characterizing the quality of the technological process is the concentration and working viscosity of the spinning solution at the outlet of the reactor. Measurement 
of these parameters is possible only in a laboratory way. Analysis of the literature $[3,8,9,14]$ and the experience of industrial operation have shown that in order to obtain a spinning solution of a given quality, it is necessary to maintain a certain temperature regime. Therefore, the reactor temperature $T_{\text {reac }}$ selected as an output parameter. The reactor temperature, in turn, is a controllable parameter, which is controlled by the temperature of the reactor jacket $T_{\text {shi. }}$.

The simulation results of the existing automatic control system show that the overshoot in the system is about $20 \%$, and the transient time is 385 seconds.

In the presence of external or parametric disturbing influences on the object (for example, a change in the vapor pressure by more than $15 \%$, a change in the concentration of the components of the reaction mixture by $10 \%$ ), the quality indicators of the transient process deteriorate significantly. In the case of a wide range of variation of these parameters, this aspect can lead the control system to an unstable state. This is due to the fact that in automatic control systems with fixed values of the parameters of the controller, the quality of the transient process changes depending on the disturbance and technological modes of the chemical reactor $[7,9]$.

Therefore, it is proposed to search for the solution of such problems using the theory of fuzzy logic, which makes it possible to operate with linguistic fuzzy statements. Thus, the problem is posed of synthesizing a robust fuzzy system for controlling the temperature regime of a chemical reactor, which is invariant to external and parametric disturbances.

\section{Solution method}

Synthesis of a fuzzy control system invariant to external and parametric disturbances.

The main stages of solving the problem are [11,12]:

1. Description of the control object and determination of its input and output parameters and disturbing influences.

2. The choice of the fuzzy inference algorithm that most fully determines the decisions made in the given conditions of the process of oil products extraction in a chemical reactor.

3. Synthesis of a fuzzy controller, which is an integral part of an intelligent controller and provides the required qualitative and quantitative indicators for controlling the temperature regime of a chemical reactor in the presence of disturbing influences.

4. Investigation of the obtained surfaces of the response of the fuzzy controller in the presence of disturbing influences and pure delay, which characterize the technological process of heat supply to the consumer.

Consider a closed system for automatic temperature control of a chemical reactor with a fuzzy logic controller (NLR) (Figure 2:).

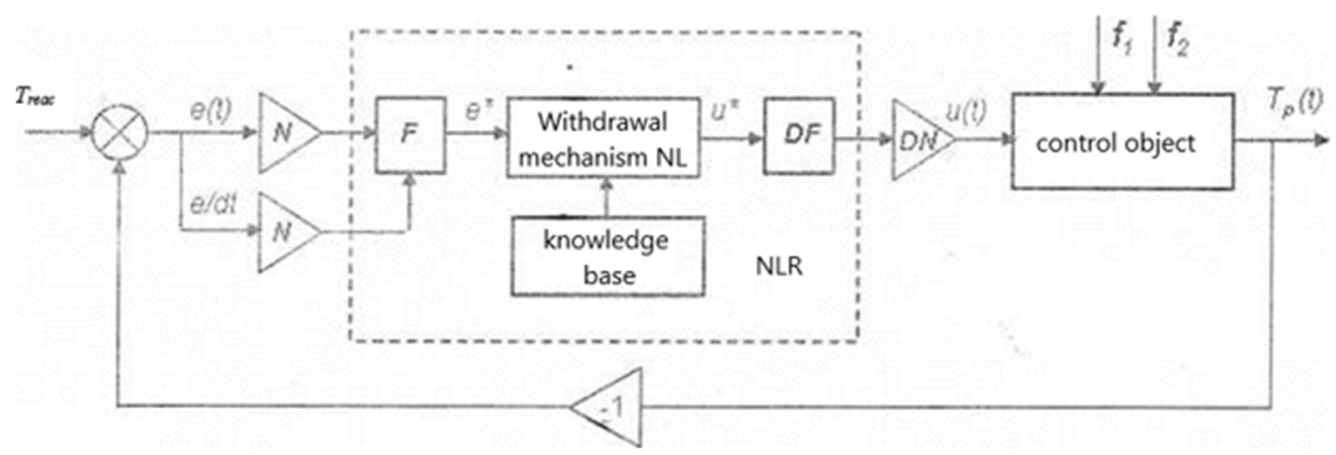

Figure 2: ATS of temperature of a chemical reactor with a fuzzy logic controller

This system differs from the existing cascade ACP with classical PI controllers in that the control loop has one single fuzzy logic controller of the MISO type with two inputs and one output. The fuzzy controller is assigned the task of developing a control action in the range of changes in the dynamic control error and its derivative with respect to its threshold values. 
According to this scheme, the input vector of the NLR $E^{*}=\left(e_{1}^{*}, e_{2}^{*}\right)$ is fuzzed using a fuzzification block $F$, then the fuzzy inference is performed in the rule base, resulting in a fuzzy output variable $u^{*}$ The translation of the values of the control vector $u^{*}$ from the fuzzy region to $u$ the clear one is carried out by the defuzzification unit $D F$.

The block $N$ is intended for preprocessing the input signal of the regulation error and its derivative:

$$
e_{t}^{N}=\left\{\begin{array}{l}
e_{i},\left|e_{i}\right| \prec e_{i}^{\max } \\
e_{i}^{\max } \operatorname{sign}\left(e_{i}\right),\left|e_{i}\right| \geq e_{i}^{\max } .
\end{array}\right.
$$

The post-processing of the output control signal is carried out by the block $D N$, where the given denormalization $u$ is solved:

$$
u=u_{N} D V=u_{N}\left|u_{\max }\right|,
$$

Where $u_{\max }$ is the maximum value of the control applied to the object.

As a rule, the NLR knowledge base contains a description of the terms of linguistic variables (LP), which must be defined in advance for each input and output variable.

For this, we introduce the following linguistic variables $e_{1}=$ ("error control", $\left.T_{e l}, E_{l}\right)$, $e_{2}=$ ("Manufacturing errors", $T_{e 2}, E_{2}$ ) and $u=$ ("Management"), where $T_{e_{j}}=\left\{T_{e_{i}}^{1}, T_{e_{t}}^{2}, \ldots T_{e_{i}}^{k}\right\}, \quad i=\overline{1, k}$, $T_{u}=\left\{T_{u}^{1}, T_{u}^{2}, \ldots T_{u}^{k}\right\}$,-term-sets of values of linguistic variables $e_{1}, e_{2}$ and $u$ with the corresponding accessory functions (FP) $T_{e_{i}}^{l}=\mu_{e i}^{l}\left(e_{i}\right), T_{u}^{l}=\mu_{1}(u), l=\overline{1, k}$, given, respectively, on the universal sets $E_{i}=\left[E_{i \min }, E_{i \max }\right]$ and $U=\left[U_{\min }, U_{\max }\right]$.

Suppose that each input and output linguistic variable $T_{x}=\left\{T_{e}, T_{e / d t}, T_{u}\right\}$ has 7 terms:

$$
T_{x}=\{" N B ", " N M ", " N S ", " Z E ", " P S ", " P M ", " P B "\} \text {, }
$$

with triangular functions accessories:

$$
\mu_{T x}(x, a, b, c)=x \leq 0\left\{\begin{array}{c}
0, \text { if } a, x \leq a \\
\frac{x-a}{b-a}, \text { if } a, a \leq x \leq b \\
\frac{c-x}{c-b}, \text { if } a, b \leq x \leq c \\
0, \text { if } a, c \leq x
\end{array}\right\}
$$

Then fuzzification results in linguistic variables:

$$
\begin{gathered}
e_{1}=\text { "Error" }=\left[\frac{\mu_{N B_{e}}(e)}{N B_{e}}, \frac{\mu_{N M_{e}}(e)}{N M_{e}}, \frac{\mu_{N S_{e}}(e)}{N S_{e}}, \frac{\mu_{Z E_{e}}(e)}{Z E_{e}}, \frac{\mu_{P S_{e}}(e)}{P S_{e}}, \frac{\mu_{P M_{e}}(e)}{P M_{e}}, \frac{\mu_{P B_{e}}(e)}{P B_{e}}\right], \\
=\left[\frac{\mu_{N B_{e} / d t}(\dot{e})}{N B_{\dot{e}}}, \frac{\mu_{N M_{\dot{e}}}(\dot{e})}{N M_{\dot{e}}}, \frac{\mu_{N S_{\dot{e}}}(\dot{e})}{N S_{\dot{e}}}, \frac{\mu_{Z E_{\dot{e}}}(\dot{e})}{Z E_{\dot{e}}}, \frac{\mu_{P S_{\dot{e}}}(\dot{e})}{P S_{\dot{e}}}, \frac{\mu_{P M_{\dot{e}}}(\dot{e})}{P M_{\dot{e}}}, \frac{\mu_{P B_{\dot{e}}}(\dot{e})}{P B_{\dot{e}}}\right] \\
e_{1}=\text { "Management" }=\left[\frac{\mu_{N B_{u}}(u)}{N B_{u}}, \frac{\mu_{N M_{u}}(u)}{N M_{u}}, \frac{\mu_{N S_{u}}(u)}{N S_{u}}, \frac{\mu_{Z E_{u}}(u)}{Z E_{u}}, \frac{\mu_{P S_{u}}(u)}{P S_{u}}, \frac{\mu_{P M_{u}}(u)}{P M_{u}}, \frac{\mu_{P B_{u}}(u)}{P B_{u}}\right],
\end{gathered}
$$

Next, we form the bases of the rules of inference of the NLR in the form:

$$
\text { if }\left(T_{e 1}^{j} \times T_{e 2}^{j}\right) \text {, TO } T_{a}^{j}, j=\overline{1,7},
$$

where $\left(T_{e 1}^{j} \times T_{e 2}^{j}\right)$ is the Cartesian product of fuzzy sets $E_{1}$ and $E_{2}$, given on the scales $E_{1}$ and $E_{2}$, with a membership function:

$$
\mu_{\left(\tau_{e 1}^{j} \times T_{e 2}^{j}\right)}\left(e_{1}, e_{2}\right)=\mu_{\tau_{e 1}^{j}}\left(e_{1}\right) \wedge \mu_{\tau_{e 2}^{j}}\left(e_{2}\right)
$$


$T_{u}^{j}$-the corresponding output fuzzy set, determined by the fuzzy relation $R^{j}=\left(T_{e 1}^{j} \times T_{e 2}^{j}\right) \times T_{u}^{j}, j=\overline{1,7}$ with the membership function:

$$
\mu_{R^{i}}\left(\left(e_{1}, e_{2}\right), u^{*}\right)=\left(\mu_{T_{e 1}^{i}}\left(e_{1}\right) \wedge \mu_{T_{e 2}^{i}}\left(e_{2}\right)\right) \wedge \mu_{T_{u}^{i}}\left(u^{*}\right) .
$$

The set of all rules corresponding to a fuzzy relation $R=\bigcup_{j=1}^{7} R^{j}$, with a membership function

$$
\mu_{R}\left(\left(e_{1}, e_{2}\right), u^{*}\right)=\vee_{j=1}^{7}\left[\left(\mu_{\tau_{e 1}^{j}}\left(e_{1}\right) \wedge \mu_{\tau_{e 2}^{j}}\left(e_{2}\right)\right) \wedge \mu_{\tau_{a}^{j}}\left(u^{*}\right)\right],
$$

defines the knowledge base of NLR and sets the law of functioning of a fuzzy system.

Thus, given the values of the input linguistic variables $T_{e 1}^{j}$ and $T_{e 2}^{j}$, the output value of the fuzzylogic controller $T_{u}^{j}$ can be determined based on the following compositional rule [13]:

with the degree of belonging:

$$
B^{j}=\left(T_{e 1}^{j} \times T_{e 2}^{j}\right) \bullet R
$$

$$
\mu_{\tau_{e}^{j}}\left(u^{*}\right)=\underset{e_{1} \in E_{1}, e_{2} \in E_{2}}{\vee}\left[\left(\mu_{\tau_{e 1}^{j}}\left(e_{1}\right) \wedge \mu_{\tau_{e 2}^{j}}\left(e_{2}\right)\right) \wedge \mu_{R}\left(e_{1}, e_{2}, u^{*}\right)\right] .
$$

In the case when the linguistic variables of the input signal $e_{1}$ and $e_{2}$ there correspond fuzzy sets, $T_{e 1}^{j}$ and $T_{e 2}^{j}$, the fuzzy set $T_{u}^{j}$ of the linguistic variable of the control signal $u^{*}$ is defined as follows:

$$
\mu_{\tau_{u}^{j}}\left(u^{*}\right)=\max _{e_{1}, e_{2}}\left\{\left[\prod_{i=1}^{n} \mu_{\tau_{e 1}^{j}}\left(e_{i}\right)\right] \cdot\left[\min _{j=1}^{m}\left[\prod_{i=1}^{n} \mu_{\tau_{e 1}^{j}}\left(e_{i}\right)\right] \cdot \mu_{\tau_{e 2}^{j}}\left(u^{*}\right)\right]\right\} .
$$

After the fuzzy inference procedure, in order to obtain the real value of the output signal of the fuzzy regulator, it is necessary to carry out the defuzzification process - translating the fuzzy value of the linguistic variable $u^{*}$ into a clear value $u$.

To do this, we use the center of gravity method [15]:

$$
u=\sum_{n=1}^{9} u_{n}^{*} \mu_{T_{u}}\left(u_{n}^{*}\right) / \sum_{n=1}^{9} \mu_{T_{u}}\left(u_{n}^{*}\right) .
$$

Considering that the membership function of a fuzzy value $T_{u}^{*}$ can be represented as:

$$
\mu_{T_{u}^{j}}(u)=\left\{\begin{array}{l}
\prod_{i=1}^{n} \mu_{T_{e 1}^{j}}\left(e_{i}\right), u=\lambda^{j} \\
0, u \neq \lambda^{j}
\end{array}\right.
$$

where $\lambda^{j}$ are discrete numerical values of the output signal, then the defining value of the output signal of the NLR at the defuzzification stage can be calculated as follows:

$$
u=\sum_{j=1}^{m} \lambda^{j}\left[\prod_{i=1}^{n} \mu_{T_{e_{i}^{j}}}\left(e_{i}\right)\right] / \sum_{j=1}^{m} \prod_{i=1}^{n} \mu_{T_{e_{i}^{j}}^{j}}\left(e_{i}\right)
$$

or

where

$$
u(\bar{e}, \bar{\lambda})=\sum_{j=1}^{m} \lambda^{j} \varsigma_{j}(\bar{e})
$$

$$
\varsigma_{j}(\bar{e})=\prod_{i=1}^{n} \mu_{T_{e_{i}^{j}}}\left(e_{i}\right) / \sum_{j=1}^{m} \prod_{i=1}^{n} \mu_{T_{e_{i}}^{j}}\left(e_{i}\right) .
$$

Considering that the basic equation of the PID controller can be written in the form 


$$
u(t)=u_{0}+K\left(e(t)+\frac{1}{T_{u}} \int_{0}^{t} e(\tau) d \tau+T_{d} \frac{d e(t)}{d t}\right),
$$

then the control law of the PID controller can be represented as a controller with a variable coefficient:

$$
K_{\text {Пид }}=K \cdot K^{*} \text {, }
$$

where $K^{*}$ is the variable part of the gain, which depends on the current value of the derivative and the integral of the control error.

This allows you to implement a fuzzy-logic PID-type controller in the form of two series-connected modules: the "NLR PD" module and the "fuzzy correction" module with adjustable coefficients $\alpha$ and $\beta$ (Figure 3:).

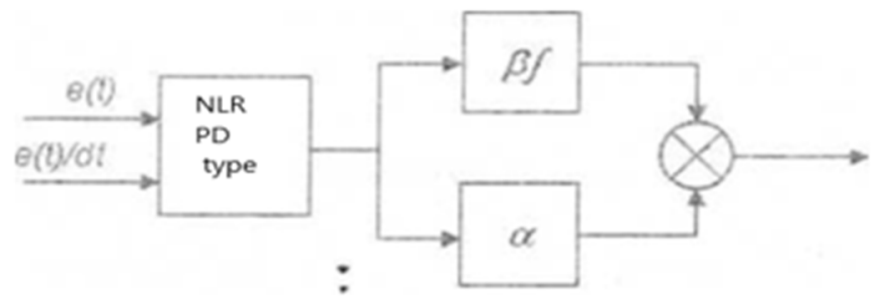

Figure 3: Structural model of a fuzzy-logic PID - controller

Thus, in the case of completeness and consistency of the base of rules of fuzzy inference, the law of functioning of the NLR can be represented as the sum of the products of two functions, determined by the type and distribution over the range of regulation of membership functions and the chosen fuzzy inference algorithm.

Based on the above theoretical considerations, we can formulate the following synthesis algorithm for a fuzzy PID controller:

1. The input and output linguistic variables of the NLR are determined, each of which contains 7 terms - sets with uniformly distributed triangular accessory functions.

2. The scaling factor and the denormalization factor of the fuzzy regulator $(\mathrm{N}, \mathrm{DN})$ are determined.

3. The bases of the rules of inference of NLR are formed in the form (1).

4. The system sequentially includes a standard linear PD with 7 terms for each LP and 49 rules, the task of which is to suppress oscillations.

5. The law of functioning of the nonlinear NLR is optimized by shifting the centers of the intermediate terms of the input LP "control error" $e_{1} ; \mu_{T_{e}}^{j}\left(x, a_{j}^{*} b_{j}^{*} c_{j}^{*}\right)$.

6. The choice of tuning parameters $\alpha$ and $\beta$, allowing the reduction of the static error.
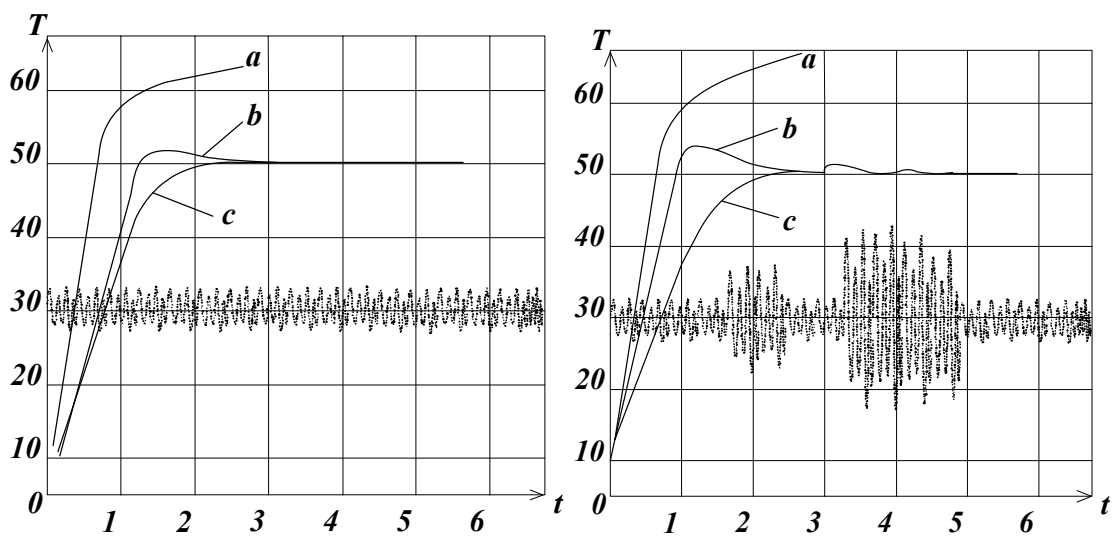

Figure 4: Transient processes in ACS system with fuzzy logic regulators under conditions when the parametrs of the control object are changed, as well as in the presence of an extrenal disturbing signal 
The considered synthesis algorithm for a fuzzy-logic PID controller is simple, since it allows the use of a standard form of description of linguistic variables and a minimum set of control rules.

\section{Results}

Figure 3: shows the results of a comparative analysis of a synthesized system of a fuzzy control system from an existing system with classical PI and PID controllers.

As can be seen from the graphs of the transient process (Figure 4) in the presence of a noisy external disturbing signal in the system and a change in its level up to $30 \%$, as well as a change in the parameters of the control object (gain $K_{1}^{O Y}, K_{2}^{O Y}$ and constant time $T_{1}^{O Y}, T_{2}^{O Y}$ ) up to $25 \%$ (in the direction of increasing and decreasing), the fuzzy system retains the stability properties.

\section{Conclusions}

Thus, on the basis of the performed computational experiments, it can be concluded that the synthesized fuzzy logic controller gives the entire automatic control system the ability to maintain the reactor temperature at a given level in the presence of external disturbances, as well as qualitatively control the polymerization process with a wide range of variation of its parameters. in time.

\section{References}

[1] R. A. Aliev, R. R. Aliev, The theory of intelligent systems, Baku, 2001.

[2] V. I. Vasiliev, B. G. Ilyasov, Intelligent control systems: Theory and practice, Moscow, 2009.

[3] B. Golding, Chemistry and technology of polymers, Moscow, 1973.

[4] V. I. Gostev, Design of fuzzy controllers for automatic control systems, Petersburg, 2011.

[5] V. N. Zakharov, S. V. Ulyanov, Fuzzy models of intelligent industrial regulators and control systems, Bulletin of the Russian Academy of Technical cybernetics 5 (1994) 35-43.

[6] I. A. Zade, The concept of a linguistic variable and its application to making approximate decisions, Moscow, 1976.

[7] I. M. Makarova, V. M. Lokhina, Intelligent automatic control systems, Moscow, 2001.

[8] V. V. Kafarov, I. N. Dorokhov, System analysis of chemical technology processes, Basics of strategy, Moscow, 1976.

[9] V. V. Kafarov, V. P. Meshalkin, Principles of development of intelligent systems in chemical technology, Bulletin of the Russian Academy 2 (1989) 409-413.

[10] A. R. Marakhimov, Neuro-fuzzy approach to restoring membership functions of values of linguistic variables, Uzbek Journal Problems of Informatics and Energy 5 (2004) 8-15.

[11] I. Pegat, Fuzzy modeling and control, Moscow, 2009.

[12] S. V. Ulyanov, L.V. Litvintseva et al., Intelligent robust control: soft computing technologies, Moscow, 2011.

[13] E. N. Mamdani, Rule-based Fuzzy Approach to the Control of Dynamic Processes II IEEE Trans, on Comput 12 (1981) 432-440.

[14] V. Rotach, The Analysis of Traditional and Fuzzy PID Rigulators, Proceeding 8-th Zittau Fuzzy Colloquium (2000) 165-172.

[15] I. Zadeh, Fuzzy logic, neural network and soft computing, Communications of the ACM 3 (1994) 30-39.

[16] Y. J. Zimmerman, Fuzzy set Theory and its applications, Second Revised Edition, 1990. 